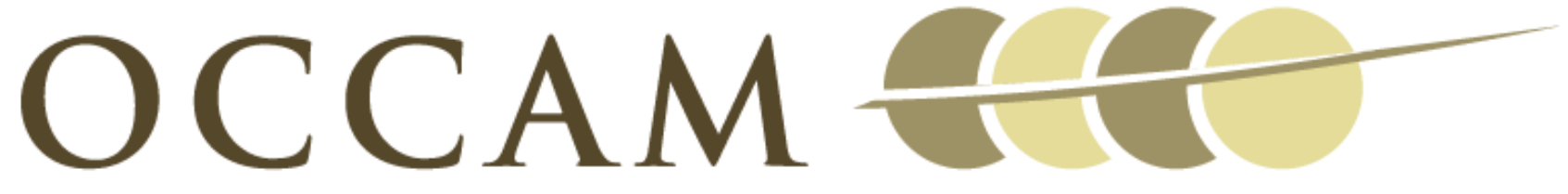

OXFORD CENTRE FOR COLLABORATIVE APPLIED MATHEMATICS

Report Number 10/46

A theory for the alignment of cortical feature maps during development

by

Paul C. Bressloff and Andrew M. Oster

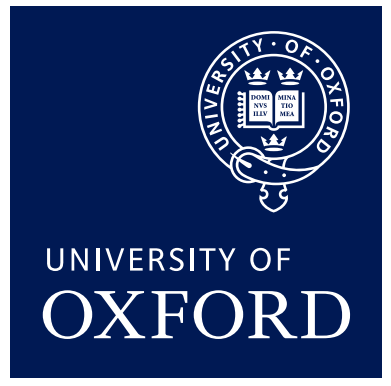

Oxford Centre for Collaborative Applied Mathematics Mathematical Institute 24 - 29 St Giles'

Oxford

OX1 3LB

England 



\title{
A theory for the alignment of cortical feature maps during development
}

\author{
Paul C. Bressloff \\ Mathematical Institute, University of Oxford, 24-29 St. Giles, Oxford OX1 3LB, UK and \\ Department of Mathematics, University of Utah, 115 S 1400 E, Salt Lake City, USA \\ Andrew M. Oster \\ Group for Neural Theory, Département des Études Cognitives, \\ École Normale Supérieure, 29, Rue d'Ulm, Paris, France
}

(Dated: July 31, 2010)

\begin{abstract}
We present a developmental model of ocular dominance column formation that takes into account the existence of an array of intrinsically specified cytochrome oxidase blobs. We assume that there is some molecular substrate for the blobs early in development, which generates a spatially periodic modulation of experience-dependent plasticity. We determine the effects of such a modulation on a competitive Hebbian mechanism for the modification of the feedforward afferents from the left and right eyes. We show how alternating left and right eye dominated columns can develop, in which the blobs are aligned with the centers of the ocular dominance columns and receive a greater density of feedforward connections, thus becoming defined extrinsically. More generally, our results suggest that the presence of periodically distributed anatomical markers early in development could provide a mechanism for the alignment of cortical feature maps.
\end{abstract}

\section{INTRODUCTION}

One of the most striking manifestations of the columnar organization of primary visual cortex are the cytochrome oxidase (CO) blobs found in both primates $[1,2]$ and cats [3]. These regions, which on average are about $0.2 \mathrm{~mm}$ in diameter and $0.5 \mathrm{~mm}$ apart, coincide with cells that are more metabolically active and hence richer in their levels of CO. The spatial distribution of $\mathrm{CO}$ blobs within cortex is correlated with a number of periodically repeating feature maps, in which local populations of neurons respond preferentially to stimuli with particular properties such as orientation, spatial frequency and ocular dominance. For example, in the macaque monkey the blobs are found at evenly spaced intervals along the center of ocular dominance (OD) columns [2], and neurons within the blobs tend to be less binocular and less orientation selective [4]. The blobs are also linked with low spatial frequency domains [5] and appear to be coincident with singularities in the orientation preference map [6]. Similar functional relationships have been shown in cat except that they tend to be weaker $[3,7]$. That is, although blobs avoid OD borders in cat visual cortex, they are not strictly aligned along the center of the columns. The arrangement of CO blobs is also reflected anatomically by the distribution of intrinsic horizontal connections, which tend to link blobs-to-blobs and interblobs-to-interblobs [4, 8, 9], and by extrinsic corticocortical connections linking blobs to specific compartments in V2 and other extrastriate areas [4, 10]. Taken together these observations suggest that the CO blobs are sites of functionally and anatomically distinct channels of visual processing.

In macaque both $\mathrm{CO}$ blobs and $\mathrm{OD}$ columns emerge prenatally, so that at birth the pattern of OD columns and their spatial relationship with blobs is adult-like. Moreover, this spatial relationship and the spacing of $\mathrm{CO}$ blobs does not appear to be influenced by visual experience $[11,12]$. In contrast, the cat's visual cortex is quite immature at birth. For example, supragranular layers of cat V1 differentiate postnatally and the blobs in these layers are normally first visible around 2 weeks of age (about one week after eye opening and one week before the critical period). This is approximately coincident with the earliest observation of OD columns in cats [13]. However, in contrast to the OD columns, altering visual experience by monocular/binocular deprivation or dark rearing does not affect the pattern of blobs, suggesting that visual experience is not necessary for the initial expression or early development of CO blobs in cat [14]. Thus, the lack of influence of visual experience on $\mathrm{CO}$ blob development in both cats and primates suggests that the blobs may reflect an innate columnar organization within superficial layers of cortex $[12,14]$. An intrinsic cortical specification of the blobs, which is distinct from the competition-driven development of cortical circuitry, could be generated by a periodic array of molecular markers. In addition to cytochrome oxidase, there are a number of other molecular markers that are arranged in a patchy fashion during early development. These include NMDA receptors [15], which play a key role in experience-dependent plasticity, serotonin receptors and synaptic zinc [16]. Interestingly, these latter markers are periodically distributed in a manner precisely complementary to cytochrome oxidase, thus tending to be located at OD column borders.

Activity-based developmental models of ocular dominance columns in visual cortex typically involve some Hebbian-like competitive mechanism for the modification of left/right eye afferents [17-19]. Intrinsic intracortical interactions consisting of short-range excitation and longer-range inhibition mediate a pattern forming instability with respect to the feedforward synaptic weights, leading to the formation of alternating left and right eye 
dominated columns. The formation of the columns is sensitive to changes in the degree of correlation among the afferents serving the two eyes. The intracortical interactions are usually taken to be homogeneous, so there is no mechanism for aligning the columns with an array of $\mathrm{CO}$ blobs. In this paper, we show how such an alignment can occur when there is a spatially periodic modulation of experience-dependent plasticity by an array of anatomical markers, which are assumed to provide an intrinsic substrate for the CO blobs early in development. The basic alignment mechanism involves mode-locking between spatial frequency components of the distribution of $\mathrm{CO}$ blobs and the emerging pattern of OD columns under the assumption of commensurability between the lateral interactions and the spacing of the molecular marker. We also show that the blobs develop a higher density of feedforward connections so that they ultimately become defined extrinsically, as they are manifested in their staining in layer $2 / 3$ of cortex. Although not considered in this work, such a spatially distributed marker might play a role in the development of patchy long-range connections within cortex that have a spacing inline with the CO blobs. Such an intrinsic patchiness of the long-range horizontal circuitry could then affect the developing formation of feature maps in V1 [20-22].

From a more general perspective, our model is an example of a hybrid model that combines both activityindependent and activity-dependent processes. Most developmental models keep these processes separate, either by positing them as alternative explanations for the same phenomenon or assuming that they contribute to different stages of development. Indeed, a common assumption is that activity-independent effects are responsible for setting up a coarse-grained version of a given feature map, which is then further refined by activity. Another example of a hybrid model occurs in the theory of topographic map formation, that is, the mapping of spatial position from eye to brain $[23,24]$. The molecular cues in this type of model are assumed to set up some sort of chemical gradient that provides a label for spatial position [25]. This should be contrasted with our model of ocular dominance formation, in which molecular cues are distributed in a periodic fashion. Such a distribution could itself arise from a diffusion-driven instability [26].

\section{DEVELOPMENTAL MODEL}

There are a variety of activity-dependent development models that generate ocular dominance columns and that can account for various visual deprivation experiments [19]. Since the basic alignment mechanism we propose is independent of the details of any particular model, we will focus on one of the simplest such models, namely the Swindale model of ocular dominance column formation [17]. This treats input layer IV of the cortex as a twodimensional sheet of neural tissue and considers competition between the synaptic densities of feedforward af- ferents from the left and right eyes. Certain care has to be taken when extending such a model to incorporate CO blobs, since the latter are found in superficial layers II/III of cortex rather than the input layer. In our simplified model, we collapse these into a single hybrid layer, motivated by the fact that there are strong vertical connections between layers early in development [27].

Let $n_{L}(\mathbf{r}, t)$ and $n_{R}(\mathbf{r}, t)$ denote the densities of left and right eye synaptic connections to a point $\mathbf{r}=(x, y)$ on the cortex at time $t$. The feedforward weights of the left and right-eye are taken to evolve according to a slightly modified version of the competitive Hebbian learning rule introduced by Swindale [17]:

$$
\begin{aligned}
\tau \frac{\partial n_{i}}{\partial t} & =\mu\left(-n_{i}+M\right) F_{N}\left(n_{i}\right) \\
& +F_{N}\left(n_{i}\right) \sum_{j=R, L} \int w_{i j}\left(\left|\mathbf{r}-\mathbf{r}^{\prime}\right|\right) n_{j}\left(\mathbf{r}^{\prime}, t\right) d \mathbf{r}^{\prime}
\end{aligned}
$$

where $i=L / R$. The logistic function $F_{N}(n)=n(N-n)$ ensures that growth terminates at a maximum weight $N$ and that the weights remain positive, that is, $0 \leq n \leq N$. The term $\mu(-n+M)$ for $\mu>0$ represents a process that tends to stabilize the uniform binocular state $n_{L}=$ $n_{R}=M$ for some constant $M$, which is identified as the initial state prior to the onset of activity-driven synaptic modification. The transition to the activity-driven phase could be modeled as a reduction in the parameter $\mu$. The lateral interaction functions $w_{L L}, w_{L R}, w_{R L}, w_{R R}$ are taken to be difference of Gaussians of the form

$$
w(r)=A \mathrm{e}^{-r^{2} / 2 \sigma_{E}^{2}}-B \mathrm{e}^{-r^{2} / 2 \sigma_{I}^{2}}
$$

where $\sigma_{E, I}$ are space constants with $\sigma_{E}<\sigma_{I}$. Same-eye interactions (RR and LL) have $A>B>0$ and oppositeeye interactions (RL and LR) have $A<B<0$. The lateral interaction functions encompass the correlation structure of the pre- and post-synaptic activities as well as the horizontal inter-laminar cortical connections (see the review [19]). Equation (1) reduces to the Swindale model if $\mu=0$ and the maximal density $N$ is spatially uniform.

We now incorporate the effects of an intrinsically defined array of CO blobs by taking $N$ to be spatially correlated with the locations of the blobs. This is motivated by the recent experimental data suggesting that the CO blobs are initially expressed in terms of some patchy anatomical marker that can modulate synaptic plasticity $[12,14,15]$. Physically the CO blob marker is directly coupled with additional thalamic input from the koniocellular pathway that innervates layers II/III in cortex, whereas the bulk of thalamic input to V1 occurs via the parvocellular and magnocellular pathways innervating layer IV. Since we are considering cortex as a compressed sheet of the cortical laminae, we consider the upperbound of synaptic weights to be non-uniform. Although the precise form of the periodic modulation is currently unknown, we postulate that the marker for the CO blobs locally enhances experience-dependent plasticity, which in the Swindale model can be interpreted as a 
local increase in $N$, that is, we assume a periodic modulation of the upperbound of the synaptic weights.

Letting $u(\mathbf{r})$ denote the patchy distribution of the $\mathrm{CO}$ marker, we set

$$
N(\mathbf{r})=\bar{N}+\kappa u(\mathbf{r})
$$

where $\bar{N}$ is a baseline level for the maximal density of feedforward afferents and $\kappa$ is a positive constant that determines the strength of the modulation. The distribution $u(\mathbf{r})$ has maxima at the CO blob centers, which are located at the sites $\boldsymbol{\ell}$ of a two-dimensional lattice $\mathcal{L}$. In order to elucidate the basic principles underlying our theory of cortical alignment, we will take $\mathcal{L}$ to be a regular planar lattice $\boldsymbol{\ell}=\left(m_{1} \boldsymbol{\ell}_{1}+m_{2} \boldsymbol{\ell}_{2}\right) d$ for integers $m_{1}, m_{2}$ with $\boldsymbol{\ell}_{1}, \boldsymbol{\ell}_{2}$ the generators of the lattice and $d$ the lattice spacing. We will subsequently show in section III that the proposed alignment mechanism is robust with respect to disorder in the distribution of $\mathrm{CO}$ blobs [12], as well as disorder in the OD column pattern. If $\theta$ is the angle between the two basis vectors $\ell_{1}$ and $\ell_{2}$, then there are three types of planar lattice distinguished by the value of $\theta$ : square lattice $(\theta=\pi / 2)$, rhombic lattice $(0<\theta<\pi / 2, \theta \neq \pi / 3)$ and hexagonal $(\theta=\pi / 3)$. The density $u(\mathbf{r})$ is assumed to be doubly periodic function on the planar lattice $\mathcal{L}$, that is, $u(\mathbf{r}+\boldsymbol{\ell})=u(\mathbf{r})$ for all lattice vectors $\ell$.

Note that there are a number of other ways in which a periodic modulation due to some chemical marker could be introduced into the developmental model (1). For example, there could be an extra additive contribution to the level of activity such that $\int w_{i j} n_{j} \rightarrow \int w_{i j} n_{j}+u$. However, this presupposes that the CO blobs have already been established as sites of enhanced activity, perhaps through an increase in the density of feedforward afferents. On the other hand, we postulate that the chemical marker modifies some aspect of synaptic plasticity, which in the Swindale model we take to be the maximum synaptic density $N$. In more detailed models of the competitive process underlying synaptic modification $[28,29]$, this could be interpreted as a modulation in the local level of neurotrophic factors; an analogous feature could also be incorporated into the correlationbased model of Miller et al. [18] by modulating the weight constraints. Yet another possibility would be to introduce a spatially periodic variation in the rate of synaptic development $\tau^{-1}$. At the linear level, all of these forms of modulation would provide a mechanism for the spatial mode-locking of the OD columns to an array of CO blobs (see section II A-2). However, in the particular case of a periodic modulation in the total density $N$, one finds that the developing pattern of OD columns naturally forms a higher density of feedforward afferents around the CO blobs, which is consistent with what is found in adult cortex. The other cases would require an additional mechanism to achieve this.

\section{A. Linear stability analysis}

Following Swindale (1980), we impose the left-right eye symmetry constraints $w_{L L}=w_{R R}=-w_{L R}=$ $-w_{R L}=w$. Equation (1) reduces to the form

$$
\begin{aligned}
\tau \frac{\partial n_{i}}{\partial t}= & \mu\left(-n_{i}+M\right) F_{N}\left(n_{i}\right) \\
& +2 F_{N}\left(n_{i}\right) \int w\left(\left|\mathbf{r}-\mathbf{r}^{\prime}\right|\right) n_{-}\left(\mathbf{r}^{\prime}, t\right) d \mathbf{r}^{\prime}
\end{aligned}
$$

where $n_{ \pm}=\left(n_{L} \pm n_{R}\right) / 2$ with $i=L / R$. It follows that the only non-zero homogeneous equilibrium is the binocular state $n_{L}^{*}=n_{R}^{*}=M$. The linear stability of the uniform binocular state can be determined by setting $n_{j}(\mathbf{r}, t)=M+\Delta n_{j}(\mathbf{r}, t)$ and expanding equation (4) to first order in $\Delta n_{j}$. In terms of the sum and difference densities $\Delta n_{ \pm}=\left(\Delta n_{L} \pm \Delta n_{R}\right) / 2$ we find that

$$
\tau \frac{\partial \Delta n_{+}(\mathbf{r}, t)}{\partial t}=-\mu \Delta n_{+}(\mathbf{r}, t) H(\mathbf{r})
$$

and

$$
\begin{aligned}
\tau \frac{\partial \Delta n_{-}(\mathbf{r}, t)}{\partial t}= & \left(-\mu \Delta n_{-}(\mathbf{r}, t)\right. \\
& \left.+\int w \mid\left(\mathbf{r}-\mathbf{r}^{\prime} \mid\right) \Delta n_{-}\left(\mathbf{r}^{\prime}, t\right) d \mathbf{r}^{\prime}\right) H(\mathbf{r})
\end{aligned}
$$

where

$$
H(\mathbf{r})=M(\bar{N}+\kappa u(\mathbf{r})-M)
$$

We will assume that $\bar{N}>M$ so that $H(\mathbf{r})$ is always positive. It follows that if $\mu>0$ then $\Delta n_{+}(\mathbf{r}, t) \rightarrow 0$ as $t \rightarrow \infty$ so that the stability of the binocular state $n_{j}(\mathbf{r})=M$ will depend on the asymptotic behavior of $\Delta n_{-}$. Writing $\Delta n_{-}(\mathbf{r}, t)=\mathrm{e}^{\lambda t / \tau} a(\mathbf{r})$, we obtain the linear eigenvalue equation

$$
\lambda a(\mathbf{r})=\left[-\mu a(\mathbf{r})+\int w\left(\left|\mathbf{r}-\mathbf{r}^{\prime}\right|\right) a\left(\mathbf{r}^{\prime}\right) d \mathbf{r}^{\prime}\right] H(\mathbf{r})
$$

The binocular state will be stable provided that all solutions of equation (8) satisfy (Re) $\lambda<0$. Note that if $\mu=0$ then the equilibrium is only marginally stable with respect to small perturbations $\Delta n_{+}$of the total density and one has to impose an additional constraint in order to specify the equilibrium uniquely [17].

\section{Homogeneous case $(\kappa=0)$}

Let us first ignore the modulatory effect of the $\mathrm{CO}$ blobs by setting $\kappa=0$ so that $H(\mathbf{r})=M(\bar{N}-M)$. Since equation (8) is homogeneous when $\kappa=0$, it has solutions of the form $a(\mathbf{r})=\mathrm{e}^{i \mathbf{k} \cdot \mathbf{r}}$ where $\mathbf{k}$ denotes two-dimensional spatial frequency and the growth factor $\lambda$ satisfies the dispersion relation

$$
\lambda=\lambda(k) \equiv[-\mu+\widehat{W}(k)] M(\bar{N}-M)
$$


with $\widehat{W}(k)$ the two-dimensional Fourier transform of $w(|\mathbf{r}|)$, that is, $\widehat{W}(k)=\int \mathrm{e}^{-i \mathbf{k} \cdot \mathbf{r}} w(|\mathbf{r}|) d \mathbf{r}$ with $k=|\mathbf{k}|$. Since $w$ satisfies equation (2), it follows that $\widehat{W}$ is a difference of Gaussians with a positive maximum at a non-zero wavenumber $k_{c}$ :

$$
\widehat{W}(k)=2 \pi\left(\sigma_{E}^{2} A \mathrm{e}^{-\sigma_{E}^{2} k^{2} / 2}-\sigma_{I}^{2} B \mathrm{e}^{-\sigma_{I}^{2} k^{2} / 2}\right)
$$

If $\widehat{W}\left(k_{c}\right)<\mu$ then the equilibrium is stable with respect to local perturbations. On the other hand, if $\widehat{W}\left(k_{c}\right)>\mu>0$ then the equilibrium is unstable due to the growth of a finite band of Fourier modes lying in some interval containing $k_{c}$ (see FIG. 1). Treating $\mu$ as a bifurcation parameter, we see that there is a primary pattern forming instability at $\mu=\mu_{c} \equiv \widehat{W}\left(k_{c}\right)$. Sufficiently close to the bifurcation point, this instability leads to the formation of a spatially periodic stripe pattern of the form $n_{-}(\mathbf{r})=A \cos (\mathbf{k} \cdot \mathbf{r}+\phi)$ with $|\mathbf{k}| \approx k_{c}$. This represents alternating left/right eye ocular dominance columns of approximate width $\pi / k_{c}$, each of which is a stripe running orthogonal to the direction of $\mathbf{k}$. Ignoring boundary effects, the direction $\arg (\mathbf{k})$ and spatial phase $\phi$ of the stripe pattern are arbitrary when $\kappa=0$, which reflects the rotational and translational symmetry of the effective lateral interactions $w$. In the case $\mu=0$ considered by Swindale [17], the band of unstable modes extends to $\infty$ such that the dynamics is far from the primary bifurcation point (see FIG. 1). The emerging pattern tends to have a more disordered stripe-like morphology, which is consistent with the pattern of OD columns found in macaque. (a)

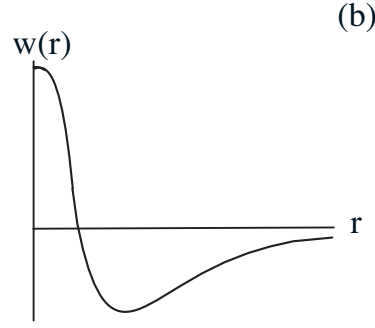

(b)

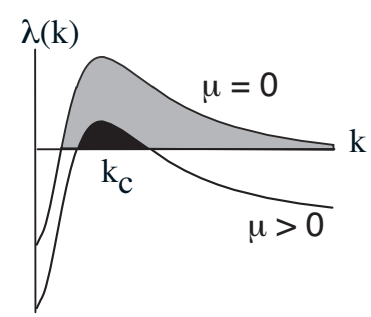

FIG. 1. (a) Difference of Gaussians lateral interaction function $w(r)$ showing short-range excitation and long-range inhibition. (b) Dispersion curve $\lambda(k)=-\mu+\widehat{W}(k)$. The shaded regions indicate the band of unstable eigenmodes around the critical wavenumber $k_{c}$ where $\widehat{W}\left(k_{c}\right)=\max _{k}\{\widehat{W}(k)\}$. Black: unstable modes for $\mu>0$. Black + gray: unstable modes for $\mu=0$.

\section{Inhomogeneous case $(\kappa>0)$}

Including the effects of the CO blobs by taking $\kappa>0$ breaks continuous rotation and translation symmetry so that the spatial phase and direction of the pattern will no longer be arbitrary. In fact, for certain critical wavevectors the pattern locks to the lattice of blobs in an analogous fashion to the pinning of flow patterns observed in periodically forced convective fluid systems [30-32]. A heuristic way to understand this pinning is to note that the factor $H(\mathbf{r})$ tends to enhance the rate of pattern growth at the blob centers, that is, at the lattice sites $\boldsymbol{\ell} \in \mathcal{L}$. Thus there is a tendency for the extrema of the resulting pattern to be located at the blobs, and this effect will be strongest when the selected pattern is commensurate with the lattice. The pinning mechanism essentially involves mode-locking between spatial frequency components of the emerging pattern of OD columns and the distribution of CO blobs. The coupling between these frequency components can be analyzed at the linear level by expanding equation (8) as a Fourier series.

In order to elucidate further the pinning mechanism, we consider the simpler case of a one-dimensional network with a periodic distribution of CO blobs: $u(x)=$ $\sum_{q} \mathcal{U}_{q} \mathrm{e}^{i q x}$ where $q=2 \pi m / d$ for integers $m$ and $d$ is the CO blob spacing. We also impose the periodic boundary condition $a(\Lambda)=a(0)$ where $\Lambda$ is the system size. In making this assumption, we are assuming that we are sufficiently far away from both the fovea and the boundary. The visual cortex is large compared to the section of cortex that we are simulating, moreover the developed visual cortex has an intrinsic periodicity, suggesting that periodic boundary conditions are a natural choice.

The one-dimensional version of equation (8) can then be analyzed by introducing the Fourier series expansion $a(x)=\Lambda^{-1} \sum_{k} A_{k} \mathrm{e}^{i k x}$ with $k=2 \pi m / \Lambda$ for integers $m$ (the periodic boundary conditions restrain the spectrum to be discrete). Substitution into equation (8) yields the eigenvalue equation (after rescaling $\lambda$ and $\kappa$ )

$$
[\lambda+\mu-\widehat{W}(k)] A_{k}=\kappa \sum_{q} \mathcal{V}_{q}(k) A_{k-q}
$$

where $\widehat{W}(k)$ is now the one-dimensional Fourier transform

$$
\begin{aligned}
\widehat{W}(k) & =\int_{-\infty}^{\infty} \mathrm{e}^{-i k x} w(x) d x \\
& =\sqrt{2 \pi} \sigma_{E} A \mathrm{e}^{-\sigma_{E}^{2} k^{2} / 2}-\sqrt{2 \pi} \sigma_{I} B \mathrm{e}^{-\sigma_{I}^{2} k^{2} / 2}
\end{aligned}
$$

and

$$
\mathcal{V}_{q}(k)=[-\mu+\widehat{W}(k-q)] \mathcal{U}_{q}
$$

At this point, we note here that equation (11) arises in previous studies examining the role of long-range periodic intra-cortical connections on pinning cortical activity patterns to the underlying cortical structure [33]. Equation (11) implies that the periodic modulation only couples together those coefficients $A_{k}, A_{k-q_{1}}, A_{k-q_{2}}, \ldots$ whose wavenumbers differ by $q_{m}=2 \pi m / d$. In other words, if we fix $k$ then $a(x)=\mathrm{e}^{i k x} b_{k}(x)$ with $b_{k}(x)$ given by the $d$-periodic function $b_{k}(x)=\sum_{q} A_{k-q} \mathrm{e}^{-i q x}$. 


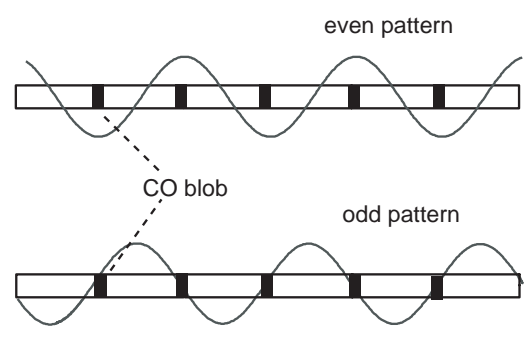

FIG. 2. Even and odd eigenmodes localized around blob and inter-blob regions.

It is generally not possible to find exact solutions of the eigenvalue equation (11). However, one can proceed by treating $\kappa$ as a small parameter and carrying out a perturbation analysis along similar lines to a recent study of spontaneous cortical activity patterns [21, 33]. Here we will present a perturbation argument for the occurrence of pinning. Let $k_{c}$ be the critical wavenumber for a pattern forming instability when $\kappa=0$. That is, $\widehat{W}\left(k_{c}\right)>\widehat{W}(k)$ for all $k>0, k \neq k_{c}$. Suppose $k_{c}$ is commensurate with the lattice spacing $d$ when $\kappa=0$, that is, $k_{c}=m \pi / d$ for some integer $m$. It follows that the pair of coefficients $A_{ \pm k}$ associated with the dominant wavenumber $k=k_{c}$ are coupled in equation (11), since $-k_{c}=k_{c}-Q$ for $Q=2 \pi m / d$. This implies that there is an approximate two-fold degeneracy, and we must treat equation (11) separately for the two cases $k$ and $k-Q$. Thus, to first order in $\kappa$, equation (11) reduces to a pair of equations for the coefficients $A_{k}$ and $A_{k-Q}$ :

$$
\left(\begin{array}{cc}
\lambda-E(k) & -\kappa \mathcal{V}_{Q}(k) \\
-\kappa \mathcal{V}_{-Q}(k-Q) & \lambda-E(k-Q)
\end{array}\right)\left(\begin{array}{c}
A_{k} \\
A_{k-Q}
\end{array}\right)=0
$$

where $E(k)=-\mu+\widehat{W}(k)+\kappa \mathcal{V}_{0}(k)$. We will assume that $u(x)$ is an even function of $x$ so that $\mathcal{U}_{Q}=\mathcal{U}_{-Q}$. Since $\widehat{W}\left(k_{c}\right)=\widehat{W}\left(k_{c}-Q\right)$ and $\widehat{W}^{\prime}\left(k_{c}\right)=0$, it follows that for $k=m \pi / d+\mathcal{O}(\kappa)$, the above matrix equation has solutions of the form (to first order in $\kappa$ )

$$
\left.\lambda_{ \pm}(k)=-\mu+\widehat{W}(k)+\kappa\left[\mathcal{V}_{0}(k) \pm \mathcal{V}_{Q}(k)\right]\right)
$$

with $A_{k-Q}= \pm A_{k}$. Thus there is a splitting into the even and odd eigenmodes

$$
a^{+}(x)=A \cos (n \pi x / d), \quad a^{-}(x)=A \sin (n \pi x / d)
$$

where $A$ is an arbitrary amplitude (at the linear level). The even and odd eigenmodes for $n=1$ are shown in FIG. 2. Note that the even mode has extrema at sites corresponding to the $\mathrm{CO}$ blobs, whereas the odd mode has extrema at sites corresponding to inter-blobs. Which of the two eigenmodes is selected by the pattern forming instability will depend on the sign of $\mathcal{V}_{Q}\left(k_{c}\right)$ for $Q=$ $2 \pi / d$. Assuming that $\mathcal{U}_{Q}>0$ then the even pattern will be selected leading to the formation of alternating left and right ocular dominance columns of width $\pi / d$ whose centers are located at the $\mathrm{CO}$ blob centers. (a)
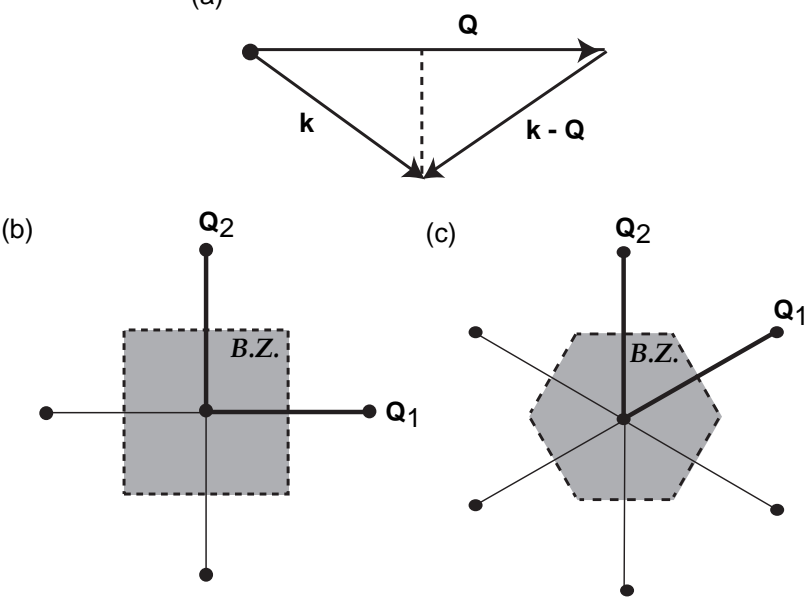

FIG. 3. (a) If $k=|\mathbf{k}-\mathbf{Q}|$ then $\mathbf{k}$ must lie on the bisector of the reciprocal lattice vector $\mathbf{Q}$. (b,c) Construction of the first Brillouin zone (shaded region) in the reciprocal square and hexagonal lattices with $\mathbf{Q}_{j}=2 \pi \widehat{\ell}_{j} / d$.

The major difference between the one dimensional and two dimensional versions of equation (8) is that in the latter case there is an additional degeneracy arising from the isotropy of the lateral interactions $w$. In principle the pattern forming instability can excite any eigenmode $\mathrm{e}^{i \mathbf{k} \cdot \mathbf{r}}$ lying on the critical circle $|\mathbf{k}|=k_{c}$ for $\kappa=0$. However, when $\kappa>0$, continuous rotation symmetry is broken by the underlying lattice of CO blobs so that the OD stripes tend to favor particular directions that reflect the discrete symmetries of the lattice. The modulation term $u(\mathbf{r})$ can be expanded as a two-dimensional Fourier series:

$$
u(\mathbf{r})=\sum_{\mathbf{q} \in \widehat{\mathcal{L}}} \mathcal{U}_{\mathbf{q}} \mathrm{e}^{i \mathbf{q} \cdot \mathbf{r}}, \quad \mathbf{q}=\sum_{j=1,2} \frac{2 \pi n_{j}}{d} \hat{\ell}_{j},
$$

where $\hat{\ell}_{j}$ are the generators of the reciprocal lattice $\widehat{\mathcal{L}}=\{\mathbf{q} \mid \mathbf{q} \cdot \boldsymbol{\ell}=0$ for all $\boldsymbol{\ell} \in \mathcal{L}\}$ with $\hat{\boldsymbol{\ell}}_{i} \cdot \boldsymbol{\ell}_{j}=\delta_{i, j}$. Such a term couples together eigenmodes with wavevectors $\mathbf{k}$ that differ by a reciprocal lattice vector $\mathbf{q}$. Extending the perturbation analysis of the one-dimensional network, it can then be shown that this leads to the following commensurability condition for the pinning of a twodimensional OD stripe pattern to the CO blobs: there exists a reciprocal lattice vector $\mathbf{Q} \in \widehat{\mathcal{L}}$ such that [21]

$$
|\mathbf{k}-\mathbf{Q}|=|\mathbf{k}|=k_{c}
$$

Geometrically this means that $\mathbf{k}$ must lie on the perpendicular bisector of the line joining the origin of the reciprocal lattice $\widehat{\mathcal{L}}$ to the lattice point $\mathbf{Q}$, see FIG. 3(a). In the theory of crystalline solids these perpendicular bisectors partition the reciprocal lattice into domains known as Brillouin zones, see FIG. 3(b,c); commensurability then requires that $\mathbf{k}$ lie on the boundary of a Brillouin zone [34]. 


\section{B. Commensurability}

So far we have assumed that the lateral interactions $w$, as given by the Mexican hat function (2), generate a stripe pattern of OD columns in which the width of each column is approximately equal to a $\mathrm{CO}$ blob spacing $d$. Is there some mechanism whereby such a matching of length scales could be achieved? An idea proposed by Bressloff [21] suggests one possibility for such a commensurability to apply based on the experimental observation (in cats and ferrets) that there exist crude patchy long-range horizontal connections early in development [35-37]. In fact, three distinct phases in the development of horizontal connections have been identified: (i) The initial appearance of long-range connections in the subplate and layer I, which could serve as a scaffold for the patchy connections subsequently found in other layers. (ii) Crude clustering of horizontal connections in superficial layers II/III (before eye opening in cat); this is not affected by dark rearing, binocular deprivation or blocking of retinal activity. (iii) An activity-dependent refinement of the patchy connections, in which the spacing of the patches is approximately conserved. Combined with the observation that the patchy connections in the adult cortex tend to link blobs in same eye OD columns [8], it follows that the spacing between horizontal patches transverse to the OD columns is approximately twice the blob spacing, and this relative spacing should also hold during early development when the blobs are first detected. One intriguing possibility is that intrinsic, patchy chemical markers mediate both the initial formation of CO blobs as well as the early development of patchy horizontal connections, thus naturally leading to a matching of length scales. The combination of the local "Mexican hat" lateral circuitry with the modulatory effects of the long-range patchy connections taken together would accentuate the effects of the lateral circuitry at the intrinsic blob spacing, that is, the Fourier mode associated with the blob spacing would be maximized.

\section{RESULTS}

As the amplitude of the selected pattern grows the dynamics will become dominated by nonlinearities arising from the logistic function $F_{N}(n)$. To what extent does the pinning of the OD pattern to the lattice of CO blobs persist when such nonlinearities are included, and is the resulting pattern stable? In order to investigate this issue, we numerically solve equation (1) for both one and two dimensional networks using periodic boundary conditions.

\section{A. Pinning in a one-dimensional network}

Consider the one-dimensional version of equation (1) with $N=1+\kappa u(x)$ and CO blob density

$$
u(x)=0.5[1+\cos (2 \pi x / d)]
$$

We choose the parameters of the Mexican hat function (2) such that $k_{c} \approx \pi / d$. Example space-time plots of the densities $n_{+}(x, t)$ and $n_{-}(x, t)$ are shown in FIG. 4 for $\kappa=0.4$. It can be seen that the initial binocular state evolves into alternating left and right eye ocular dominance columns whose centers align with the $\mathrm{CO}$ blobs. A periodic variation in the total density of feedforward afferents also emerges, which can be understood as follows. If a point $x$ lies within a left eye ocular dominance column we expect $n_{L}(x, t) \rightarrow \bar{N}+\kappa u(x)$ and $n_{R}(x, t) \rightarrow 0$ as $t \rightarrow \infty$, whereas we expect the opposite to occur if $x$ lies within a right eye column. This suggests that there will be a periodic variation in the total density of feedforward afferents such that $n_{+}(x) \approx(\bar{N}+\kappa u(x)) / 2$. Thus the pinning mechanism also provides a means for generating a higher density of feedforward afferents to the CO blobs so that they ultimately become defined extrinsically. This is consistent with what is found in the mature cortex, where the CO blobs in superficial layers are sites of enhanced metabolic activity resulting from a higher density of afferent inputs. (For example, in macaque the $\mathrm{CO}$ blobs receive additional, koniocellular inputs directly from the LGN). When interpreting our numerical results, however, it is important to remember that we have collapsed superficial layers II/II and input layer IV into a single effective layer. Thus the ocular dominance columns shown in FIG. 4 are more consistent with input layer IV, since each column is predominately monocular. The variation in left/right eye dominance is smoother in superficial layers so that there is a more gradual transition from monocular to binocular domains. On the other hand, the periodic variation in the total synaptic density is more consistent with superficial layers where the $\mathrm{CO}$ blobs are located, since the total density of synapses is approximately uniform in the input layer IV.

How robust is the pinning mechanism with respect to parameter variations in the strength $\kappa$ of the periodic modulation or the mismatch $\Delta_{k}=d k_{c} / \pi-1$ between the pattern wavelength and the blob spacing? In order to answer this question we introduce the following index for the degree of pinning:

$$
\chi=1-\frac{4}{P d}\left\langle\sum_{p=1}^{P}\left|x_{p}^{O D}-x_{p}^{B}\right|\right\rangle
$$

where $x_{p}^{O D}$ denotes the center of the $p t h$ OD column, $p=1, \ldots, P$, and $x_{p}^{B}$ is the location of the nearest $\mathrm{CO}$ blob center. The angular brackets $\langle$.$\rangle denote averag-$ ing over trials with random initial conditions about the binocular state. Thus $\chi=1$ for perfect pinning, $\chi=-1$ for perfect anti-pinning and $\chi=0$ for a random alignment (in which $\left|x_{p}^{O D}-x_{p}^{B}\right|$ is uniformly distributed over 
(a)

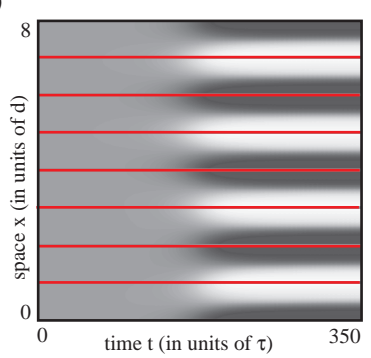

(b)

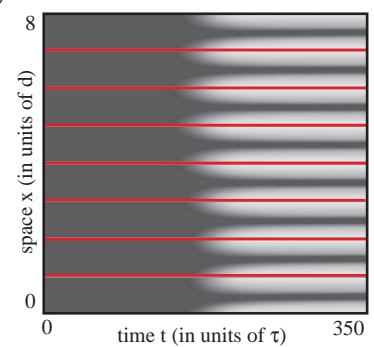

FIG. 4. (Color online) One-dimensional pinning of OD columns to a periodic array of CO blobs. (a) Space-time plot of the density $n_{-}(x, t)$ obtained by numerically solving a one-dimensional version of equation (4) for $N=1+0.5 \kappa[1+$ $\cos (2 \pi x / d)]$ with $\kappa=0.4, \mu=0.08 \approx \mu_{c}$ and a network of size $\Lambda=8 d$. The parameters of the Mexican hat function (2) are chosen to be $A=1.8, B=1.0, \sigma_{E}=0.29 d, \sigma_{I}=0.72 d$ so that $k_{c} \approx \pi / d$. The initial binocular state (grey) evolves into alternating left and right eye ocular dominance columns (colored white and black, respectively) whose centers align with the centers of the CO blobs (indicated by red horizontal lines). (b) Corresponding plot of $n_{+}(x, t)$ showing the emergence of a periodic variation in the total density of feedforward afferents (white represents a high density of synaptic drive).

the interval $[0, d / 2])$. In FIG. 5(a) we plot $\chi$ as a function of $\kappa$ in the case $\mu \approx \mu_{c}$. It can be seen that the degree of pinning tends to grow with the strength of modulation $\kappa$ for fixed $k_{c}=\pi / d$ although, even for quite large values of $\kappa$, perfect pinning does not occur. The latter is due to the occurrence of phase defects in a small number of trials; the existence of phase defects is a well known phenomenon in the theory of spatial pattern formation [38]. In our particular case, a phase defect corresponds to a localized region over which the centers of the ocular dominance columns are gradually shifted relative to the $\mathrm{CO}$ blob centers by a net spatial phase equal to a single blob spacing. An example is shown in FIG. 5(b) for a larger system size where the phase defect can be seen more clearly.

Now suppose that we take $\mu=0$ as in the Swindale model [17]. Since the system is operating far from the bifurcation point when $\mu=0$, it is outside the perturbative regime considered in section II A. In particular, we find that $\kappa=\mathcal{O}(1)$ in order for good pinning to occur, see FIG. 6(a). Another interesting feature also emerges for larger $\kappa$ as illustrated in FIG. 6(b). Here we plot the degree of pinning $\chi$ as a function of $\Delta_{k}$ for fixed $\kappa=1$ (black dots). As one might expect from the linear analysis of section II B, pinning is maximal when $\Delta_{k} \approx 0$ or $k_{c} \approx \pi / d$, that is, when the pattern wavelength is commensurate with the $\mathrm{CO}$ blob spacing.

As we have already commented, the actual distribution of $\mathrm{CO}$ blobs is not given by a perfect lattice. We find that the pinning mechanism is robust to certain levels of irregularity in the distribution of CO blobs. One way to introduce a degree of irregularity in the one-dimensional
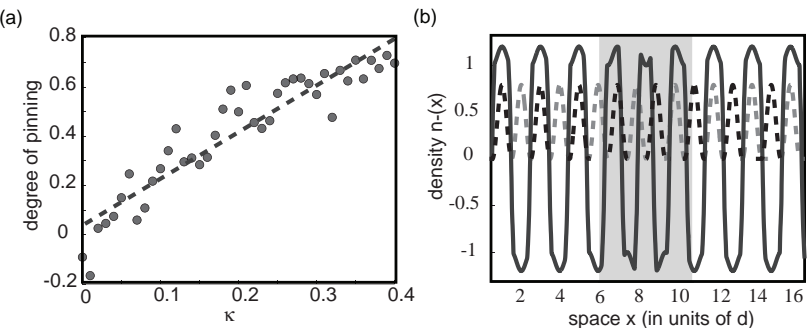

FIG. 5. (a) Plot of degree of pinning $\chi$ as a function of the modulation strength $\kappa$ for $k_{c}=\pi / d$ and $\mu=0.08$ taken over 45 trials with white noise about a binocular state. (b) Onedimensional OD pattern with a spatial phase defect. The steady-state density $n_{-}(x)$ is plotted as a function of $x$ for $\Lambda=16 d$. Other parameter values are as in FIG. 4. It can be seen that pinning occurs except within a transition region (shaded in gray) where the OD columns are gradually shifted by an additional spatial phase equal to a single blob spacing. The variation in the CO blob density $u(x)$ is shown by the alternating light and dark dashed curve. The maxima of the density $n_{-}(x)$ shift from the dark to the light peaks of $u(x)$ as $x$ increases.
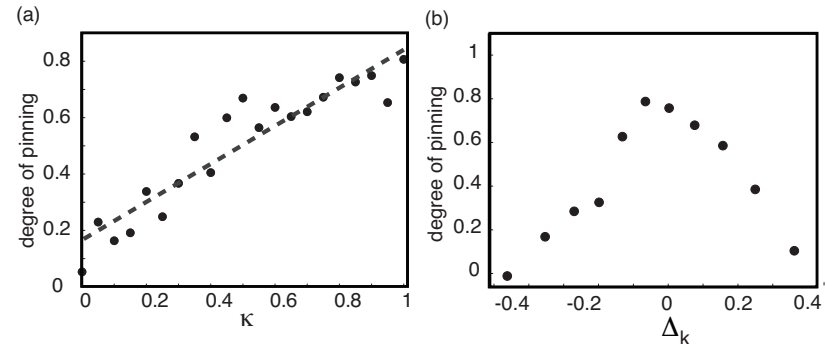

FIG. 6. (a) Plot of degree of pinning $\chi$ as a function of the modulation strength $\kappa$ for $k_{c}=\pi / d$. (b) Plot of degree of pinning $\chi$ as a function of the wavelength mismatch $\Delta_{k}=$ $d k_{c} / \pi-1$ for $\kappa=1.0$. Black dots show pinning with respect to OD centers. Weight parameters are as in FIG. 4 except that $\mu=0$. Averages were performed over 40 trials.
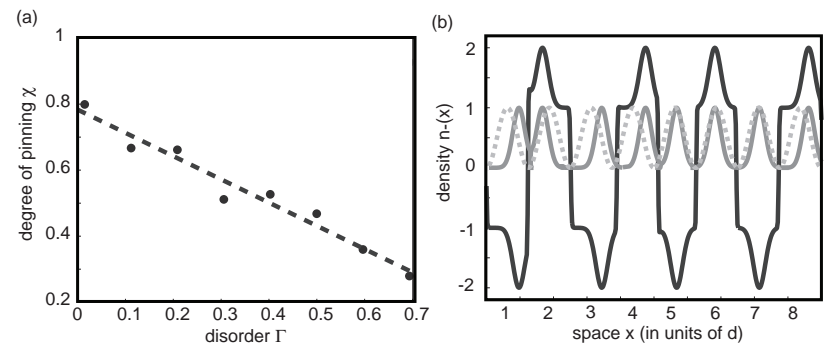

FIG. 7. (a) Plot of degree of pinning $\chi$ versus degree of irregularity $\Gamma$ in distribution of $\mathrm{CO}$ blobs as given by equation (20). Here $\kappa=1.0, \mu=0, k_{c}=\pi / d$. (b) One-dimensional OD pattern (black curve) pinning to an irregular distribution $(\Gamma=0.7)$ of CO blobs (solid gray curve). The corresponding regular distribution of spacing $d$ is shown by the dashed gray curve. Same parameter values as (a) with $\Gamma=0.7$. 
model is by taking the CO blob density to be of the form

$$
u(x)=\sum_{p=1}^{P} \exp \left(-\left(x-x_{p}\right)^{2} / 2 \gamma^{2}\right)
$$

where $\gamma$ determines the patch size (assumed to be smaller than $d$ ). The center $x_{p}$ of the $p$ th blob is taken to be $x_{p}=p d+\Gamma \xi_{p}$ with $\xi_{p}$ a random variable generated from a uniform distribution over the interval $[-0.5,0.5]$. Thus $\Gamma$ is a measure of the degree of disorder. In FIG. 7(a) we plot the degree of pinning $\chi$ as a function of $\Gamma$. We see that pinning persists for irregular lattices with the most monocular regions lining up with the blobs as illustrated in FIG. 7(b). Again our model predicts a shift between the center of the OD column and the most monocular region, depending on the degree of disorder and the size of $\kappa$; for smaller values of $\kappa$ this shift would be negligible. From an experimental perspective, the current finding in macaque is that the $\mathrm{CO}$ blobs tend to lie in the centers of OD columns and also tend to coincide with the most monocular cell populations. As far as we are aware, the possibility of a statistically significant difference between these two aspects has not been investigated.

\section{B. Pinning in a two-dimensional network}

All of the above observations extend to the more realistic case of two-dimensional networks. In FIG. 8 we show examples of two-dimensional OD patterns obtained by numerically solving equation (4) for $\mathrm{CO}$ blobs on a square lattice. We take $N=1+\kappa u(x, y)$ with

$$
u(x, y)=0.25[2+\cos (2 \pi x / d)+\cos (2 \pi y / d)]
$$

representing the two-dimensional distribution of $\mathrm{CO}$ blobs and $\kappa=1.0, \mu=0$. The parameters of the weight distribution $w(r)$ given by equation (2) are chosen so that $k_{c} \approx \pi / d$. The resulting OD stripes tend to be oriented in directions corresponding to symmetries of the lattice and the centers of the OD columns tend to coincide with CO blob centers.

Interestingly, pinning occurs even though the patterns are quite irregular with a number of defects and branches. Pinning also persists when there is a certain level of disorder in the two-dimensional distribution of the blobs. This is illustrated in FIG. 9 for the Gaussian CO blob distribution

$$
u(x, y)=\sum_{p} \mathrm{e}^{\left(-\left(x-x_{p}\right)^{2} / 2 \gamma^{2}\right)} \mathrm{e}^{\left(-\left(y-y_{p}\right)^{2} / 2 \gamma^{2}\right)}
$$

Here the center $\left(x_{p}, y_{p}\right)$ of the $p$ th blob is given by $x_{p}=$ $p d+\Gamma \xi_{p}, y_{p}=p d+\Gamma \eta_{p}$ with $\xi_{p}, \eta_{p}$ random variables generated from a uniform distribution over the interval $[-0.5,0.5]$. As in the one-dimensional case, the CO blobs are strongly correlated with the most monocular regions of the OD column. Similar results also hold for regular or irregular rectangular and hexagonal lattices.
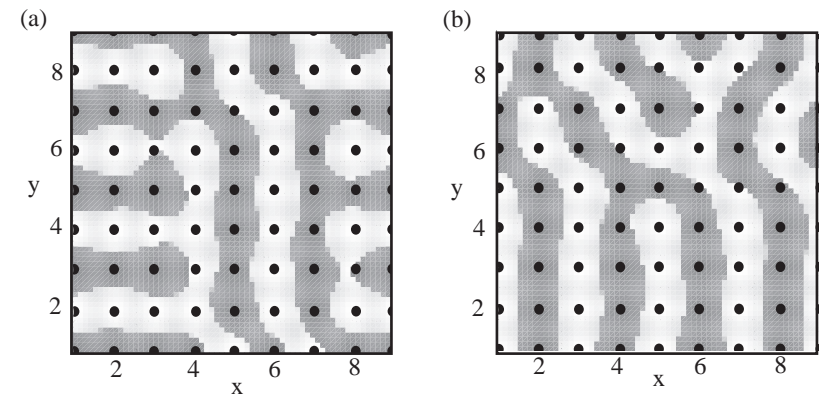

FIG. 8. Two-dimensional pinning of OD columns to a square lattice of $\mathrm{CO}$ blobs with $N=1+\kappa u(x, y)$ and $u(x, y)=0.25[2+\cos (2 \pi x / d)+\cos (2 \pi y / d)]$. We take $\mu=0$, $\kappa=1$ and consider a radial weight distribution (2) with $A=3.8, B=3.3, \sigma_{e}=0.51 d, \sigma_{i}=0.64 d$ such that $k_{c} \approx \pi / d$ where $d$ is the blob spacing. The two examples correspond to different initial conditions obtained by introducing small random perturbations of the uniform binocular state. For visualization purposes the color scale was chosen so that white corresponds to left-eye dominance and grey corresponds to right-eye dominance, with the lattice shown in black.

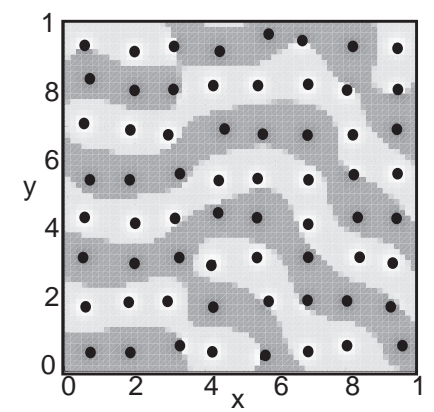

FIG. 9. Two-dimensional pinning of OD columns to an irregular lattice of CO blobs. Distribution of CO blobs is given by equation (22) with $\Gamma=0.3$.

Since the CO blobs are intrinsically defined in our model, it follows that their spatial relationship with the ocular dominance columns is robust with respect to interventions that mimic the effects of monocular deprivation during the critical period in macaque [11]. Following Swindale [17, 19], monocular deprivation can be modeled as a temporary reduction in the strength of the central part of the lateral interaction function (2) originating from one eye. In the case of a monocularly deprived right eye, this would involve a reduction in $A_{R R}$ and $A_{R L}$, shown in FIG. 10(a). Although the width of the stripes associated with the deprived eye shrink at the expense of the non-deprived eye, the $\mathrm{CO}$ blobs still emerge as the sites of highest density afferents associated with the most monocular parts of the OD columns. Finally, one can also introduce a left/right eye asymmetry that tends to produce OD patterns more consistent with cat, that is, circular islands of OD columns from one eye 
embedded in a complementary pattern of OD columns from the other eye [19], shown in FIG. 10(b). There is still a tendency for the blobs to coincide with the centers of the OD columns but the patchy nature of the OD columns leads to a weaker correspondence than occurs for the stripe patterns.
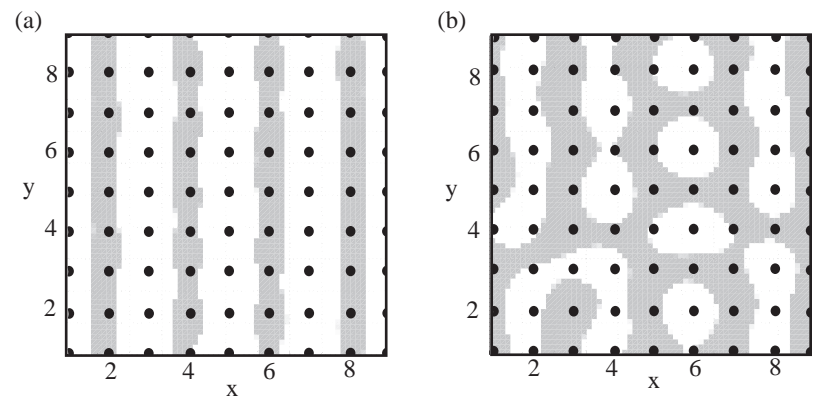

FIG. 10. Examples of pinning to the CO blob lattice in the cases of monocular deprivation (MD) and "cat-like" conditions, shown in (a) and (b), respectively. In both (a) and (b), the $\mathrm{CO}$ blob lattice and the primary weight distribution are the same as in Fig. 8. In (a), we simulate MD conditions by reducing the excitation level of the deprived eye to 60 percent its original value. The area that the non-deprived eye then innervates 67 percent of V1. In (b), since the contralateral visual input is dominant in early cat development, so to simulate cat-like conditions both excitation and inhibition levels of the ipsi-lateral drive are reduced to 60 percent their original values for the initial phase of development, and $\kappa$ is taken to be $\kappa=0.1$. For visualization purposes the color scale was chosen so that white corresponds to left-eye dominance and grey corresponds to right-eye dominance, with the lattice shown in black.

\section{DISCUSSION}

In this paper we have presented a simple model of ocular dominance (OD) column formation that incorporates the effects of a spatially periodic modulation in synaptic plasticity. We have suggested that such a modulation could arise from a distribution of patchy chemical markers early in development, corresponding to an array of intrinsically defined cytochrome oxidase (CO) blobs. Spatial coupling between the emerging pattern of OD columns and the periodic markers provides a mechanism for aligning the OD columns with the CO blobs. Such a mechanism is robust with respect to a certain degree of disorder in the distribution of the blobs as well as irregularities (defects and branches) in the OD pattern.

An obvious extension of our work is to investigate the possible role of CO blobs in the alignment of other cortical feature maps. For example, one could carry out an analogous modification of Swindale's model for the development of orientation columns [39]. The latter model is formulated in terms of a complex-valued function $z(\mathbf{r})$, with $\arg z(\mathbf{r})$ specifying the orientation preference at $\mathbf{r}$ (restricted to lie in the interval $[0, \pi)$ ) and $|z(\mathbf{r})|$ determining the associated orientation selectivity. The evolution equation for $z$ takes the form (for $\mu=0$ )

$$
\frac{\partial z}{\partial t}=\int w\left(\mathbf{r}-\mathbf{r}^{\prime}\right) z\left(\mathbf{r}^{\prime}\right) d \mathbf{r}^{\prime} F(z)
$$

with $F(z)=N-|z|$. The effects of a CO blob distribution could be incorporated into the model by taking $N$ to satisfy equation (3). One could then investigate to what extent the resulting orientation pinwheels align with the CO blobs as suggested by optical imaging data [6]. Swindale's model for the joint development of ocular dominance and orientation columns [40] could be modified along similar lines. The possible role of $\mathrm{CO}$ blobs in the joint alignment of OD and orientation columns has also been briefly reported in a computational study by Jones and Leyton-Brown [41].

It is possible that the coalignment of multiple feature maps could occur in the absence of a spatially periodic distribution of molecular markers. Indeed, it has previously been demonstrated that OD and orientation columns can develop simultaneously, such that orientation pinwheels align with the centers of the OD columns and iso-orientation contours cross OD column boundaries orthogonally [40, 42]. The inclusion of an additional feedforward label for the distribution of CO blobs (perhaps by taking into account the koniocellular pathway that feeds directly to the $\mathrm{CO}$ blobs in macaque) could result in the simultaneous alignment of the OD columns, the orientation preference map and the CO blobs. In contrast to the mode-locking mechanism proposed in this paper, such an alignment would arise spontaneously through the competitive process that drives the development of the multiple feature maps. There are, however, two potential difficulties with the latter approach. First, the coalignment of multiple feature maps tends to be sensitive to the choice of parameter values [42]. Second, it does not take into account the experimental finding that the spatial distribution of the CO blobs appears early in development and is not affected by activity $[12,14]$. We also note that an abstract approach of using elastic neural nets can produce results in line with cortical feature maps seen in V1 [43], however here we have attempted to develop a biologically-inspired mathematical model in line with the anatomy.

Finally, it is tempting to argue that a spatially periodic distribution of molecular markers early in development orchestrates the coalignment of multiple feature maps in order to achieve a modular architecture. This is consistent with computational models that treat V1 as a system of coupled hypercolumns [44, 45]. Each hypercolumn processes local features of an image by carrying out some form of local Fourier decomposition, say; the results of these local computations are then correlated within V1 through long-range horizontal connections and transmitted to extrastriate areas for further processing. It would be interesting to determine whether or not animals without such modular architectures still have periodically dis- 
tributed markers.

\section{ACKNOWLEDGMENTS}

This publication was based on work supported in part by the NSF (DMS-0209824) and by Award No KUK-C1-
013-4 made by King Abdullah University of Science and Technology (KAUST). PCB was also partially supported by the Royal Society-Wolfson Foundation.
[1] J. C. Horton and D. H. Hubel. Regular patchy distribution of cytochrome oxidase staining in primary visual cortex of macaque monkey. Nature, 292:762-764, 1981.

[2] J. C. Horton. Cytochrome oxidase patches: a new cytoarchitectonic feature of monkey visual cortex. Phil. Trans. R. Soc. Lond. B, 304:199-253, 1984.

[3] K. M. Murphy, D. G. Jones, and R. C. Van Sluyters. Cytochrome oxidase blobs in cat primary visual cortex. Neurosci., 15:4196-4208, 1995.

[4] M. S. Livingstone and D. H. Hubel. Specificity of intrinsic connections in primate primary visual cortex. $J$. Neurosci., 4:2830-2835, 1984.

[5] R. B. Tootell, M. S. Silverman, S. L. Hamilton, E. Switkes, and R. L. De Valois. Functional anatomy of macaque striate cortex. V. spatial frequency. J. Comp. Neurol., 8:1610-1624, 1988.

[6] K. Obermayer and G. G. Blasdel. Geometry of orientation and ocular dominance columns in monkey striate cortex. J. Neurosci., 13:4114-4129, 1993.

[7] D. Shoham, M. Hubener, S. Schulze, A. Grinvald, and T. Bonhoeffer. Spatio-temporal frequency domains and their relation to cytochrome oxidase staining in cat visual cortex. Nature, 385:529-533, 1997.

[8] T. Yoshioka, G. G. Blasdel, J. B. Levitt, and J. S. Lund. Relation between patterns of intrinsic lateral connectivity, ocular dominance, and cytochrome oxidase-reactive regions in macaque monkey striate cortex. Cerebral Cortex, 6:297-310, 1996.

[9] N. H. Yabuta and E. M. Callaway. Cytochrome-oxidase blobs and intrinsic horizontal connections of layer $2 / 3$ pyramidal neurons in primate V1. Visual Neurosci., 15:1007-1027, 1998.

[10] L. C. Sincich and J. C. Horton. Divided by cytochrome oxidase: A map of the projections from V1 to V2 in macaques. Science, 295:1734-1737, 2002.

[11] J. C. Horton and D. R. Hocking. Timing of the critical period for plasticity of ocular dominance columns in macaque striate cortex. J. Neurosci., 17:3684-3709, 1997.

[12] K. M. Murphy, D. G. Jones, S. B. Fenstemeaker, V. D. Pegado, L. Kiorpes, and J. A. Movshon. Spacing of cytochrome oxidase blobs in visual cortex of normal and strabismic monkeys. Cerebral Cortex, 8:237-244, 1998.

[13] M. C. Craire, J. C. Horton, and M. P. Stryker. Emergence of ocular dominance columns in cat visual cortex by 2 weeks of age. J. Comp. Neurol., 430:235-249, 2001.

[14] K. M. Murphy, K. R. Duffy, D. G. Jones, and D. E. Mitchell. Development of cytochrome oxidase blobs in visual cortex of normal and visually deprived cats. Cerebral Cortex, 11:122-135, 2001.

[15] C. Trepel, D. R. Duffy, V. D. Pegado, and K. M. Murphy. Patchy distribution of NMDAR1 subunit immunoreactivity in developing visual cortex. J. Neurosci., 18:3404-
$3415,1998$.

[16] R. H. Dyck and M. S. Cynader. An interdigitated columnar mosaic of cytochrome oxidase, zinc and neurotransmitter-related molecules in cat and monkey visual cortex. Proc. Natl. Acad. Sci. USA, 90:9066-9069, 1993.

[17] N. V. Swindale. A model for the formation of ocular dominance stripes. Proc. R. Soc. B, 208:243-264, 1980.

[18] K. D. Miller, J. B. Keller, and M. P. Stryker. Ocular dominance column development: analysis and simulation. Science, 245(4918):605-615, 1989.

[19] N. V. Swindale. The development of topography in the visual cortex: a review of models. Network: Computation in Neural Systems, 7:161-247, 1996.

[20] H. Z. Shouval, D. H. Goldberg, J. P. Jones, M. Beckerman, and L. N. Cooper. Structured long-range connections can provide a scaffold for orientation maps. $J$. Neurosci., 20(3):1119-1128, 2000.

[21] P. C. Bressloff. Spatially periodic modulation of cortical patterns by long-range horizontal connections. Physica D, 185:131-157, 2003.

[22] J. J. Wright, D. M. Alexander, and P. D. Bourke. Contribution of lateral interactions in V1 to organization of response properties. Vision Res., 46:2703-2720, 2006.

[23] V. A. Whitelaw and J. D. Cowan. Specificity and plasticity of retinotectal connections: a computational model. J. Neurosci., 1:1369-1387, 1981.

[24] C. Weber, H. Ritter, J. D. Cowan, and K. Obermayer. Development and regeneration of the retinotectal map in goldfish: a computational study. Phil. Trans. B, 352:1603-1623, 1997.

[25] D. Willshaw and D. Price. Modeling Neural Development, chapter Models of topographic map formation, pages 213-244. MIT Press, 2003.

[26] A. M. Turing. Chemical basis of morphogenesis. Phi. Trans. Roy. Soc. B (London), 237:37-72, 1952.

[27] E. M. Callaway. Prenatal development of layer specific local circuits in primary visual cortex of the macaque monkey. J. Neurosci., 18:1505-1527, 1998.

[28] A. E. Harris, G. B. Ermentrout, and A. L. Small. A model of ocular dominance column development by competition for trophic factor: effects of excess trophic factor with monocular deprivation and effects of antagonist of trophic factor. J. Comp. Neurosci., 8:227-250, 2000.

[29] A. van Ooyen and R. R. Ribchester. Modeling Neural Development, chapter Competition in the development of nerve connections, pages 183-212. MIT Press, 2003.

[30] M. Lowe and J. P. Gollub. Solitons and the commensurate-incommensurate transition in a convective nematic fluid. Phys. Rev. A, 31:3893-3896, 1985.

[31] P. Coullet. Commensurate-incommensurate transition in nonequilibrium systems. Phys. Rev. Lett., 56:724-727, 
1986.

[32] D. Walgraef. Spatio-Temporal Pattern Formation. Springer-Verlag, Berlin, 1997.

[33] P. C. Bressloff. Bloch waves, periodic feature maps and cortical pattern formation. Phys. Rev. Lett., 89:088101, 2002.

[34] N. W. Ashcroft and N. D. Mermin. Solid state physics. Saunders College Publishing, 1976.

[35] E. M. Callaway and L. C. Katz. Experience and refinement of clustered horizontal connections in cat striate cortex. J. Neurosci., 10:1134-1154, 1990.

[36] E. S. Ruthazer and M. P. Stryker. The role of activity in the development of long-range horizontal connections in area 17 of the ferret. J. Neurosci., 16:7253-7269, 1996.

[37] C. Chiu and M. Weliky. Relationship of correlated spontaneous activity to functional ocular dominance columns in the developing visual cortex. Neuron, 35:1123-1134, 2002.

[38] P. Bak. Commensurate phases, incommensurate phases and the devil's staircase. Rep. Prog. Phys., 45:587-627, 1982.

[39] N. V. Swindale. A model for the formation of orientation columns. Proc. Roy. Soc. Lond. B, 215:211-230, 1982.
[40] N. V. Swindale. A model for the coordinated development of columnar systems in primate striate cortex. Biol. Cybern., 66:217-230, 1992.

[41] D. G. Jones and K. Leyton-Brown. The role of cytochrome-oxidase blobs in the development of ocular dominance and orientation maps. Soc. Neurosci. Abstr., 24:813, 1998.

[42] E. Erwin and K. D. Miller. Correlation-based development of ocularly matched orientation and ocular dominance maps: determination of required input activities. J. Neurosci., 18:9870-9895, 1998.

[43] M. Á. Carreira-Perpĩná, R. J. Lister, and G. J. Goodhill A computational model for the development of multiple maps in primary visual cortex Cerebral Cortex, 15:12221233, 2005.

[44] S. LeVay and S. B. Nelson. The neural basis of visual function, chapter Columnar organization of the visual cortex, pages 266-315. CRC Press, 1991.

[45] J. S. Lund, A. Angelucci, and P. C. Bressloff. Anatomical substrates for functional columns in macaque monkey primary visual cortex. Cerebral Cortex, 12:15-24, 2003. 



\section{RECENT REPORTS}

22/10 Fat vs. thin threading approach on GPUs: application to stochastic simulation of chemical reactions

Klingbeil

Erban

Giles

Maini

23/10 Asymptotic analysis of a system of algebraic equations arising in

Hall dislocation theory

Chapman

Ockendon

25/10 Preconditioning for Allen-Cahn Variational Inequalities with Non-

Blank Local Constraints

Sarbu

Stoll

26/10 On an evolution equation for sand dunes

Ellis

Fowler

27/10 On Liquid Films on an Inclined Plate

Benilov

Chapman

McLoed

Ockendon

Zubkov

28/10 An a posteriori error analysis of a mixed finite element Galerkin

Memon approximation to second order linear parabolic problems

Nataraj

Pani

29/10 A Priori Error Estimates for Semidiscrete Finite Element Approx-

Goswami imations to Equations of Motion Arising in Oldroyd Fluids of Order One

30/10 The Landau-de Gennes theory of nematic liquid crystals: Uniaxiality versus Biaxiality

31/10 The Radial-Hedgehog Solution in Landau-de Gennes' theory

Majumdar

32/10 Nonlinear instability in flagellar dynamics: a novel modulation mechanism in sperm migration?

Gadelha

Gaffney

Smith

Kirkman-Brown

33/10 Error bounds on block GaussSeidel solutions of coupled multiphysics problem

Whiteley

Gillow

Tavener

Walter

34/10 A random projection method for sharp phase boundaries in lattice Boltzmann simulations

Reis

Dellar

35/10 Regularized Particle Filter with Langevin Resampling Step

Duan

Farmer

Moroz

36/10 Sequential Inverse Problems Bayesian Principles and the Logistic

Duan Map Example

Farmer

Moroz

37/10 Circumferential buckling instability of a growing cylindrical tube 
38/10 Preconditioners for state constrained optimal control problems

Stoll with Moreau-Yosida penalty function

Wathen

39/10 Local synaptic signaling enhances the stochastic transport of

Newby motor-driven cargo in neurons

Bressloff

40/10 Convection and Heat Transfer in Layered Sloping Warm-Water Aquifer

McKibbin

Hale

Style

41/10 Optimal Error Estimates of a Mixed Finite Element Method for

Goswami

Parabolic Integro-Differential Equations with Non Smooth Initial

Pani

Data

Yadav

42/10 On the Linear Stability of the Fifth-Order WENO Discretization

Motamed

Macdonald

Ruuth

43/10 Four Bugs on a Rectangle

Chapman

Lottes

Trefethen

44/10 Mud peeling and horizontal crack formation in drying clay

Style

Peppin

Cocks

45/10 Binocular Rivalry in a Competitive Neural Network with Synaptic

Kilpatrick Depression

Bressloff

Copies of these, and any other OCCAM reports can be obtained from:

Oxford Centre for Collaborative Applied Mathematics Mathematical Institute

24 - 29 St Giles'

Oxford

OX1 3LB

England

www.maths.ox.ac.uk/occam 